\title{
Computed Tomography and Radiographic Lymphography of the Thoracic Duct by Subcutaneous or Submucosal Injection
}

\author{
Kenji ANDO $^{1)}$, Keiji KAMIJYOU ${ }^{1,2)}$, Ken HATINODA ${ }^{1)}$, Shinji SHIBATA ${ }^{1,3)}$, Takuo SHIDA ${ }^{4)}$ and \\ Masao ASARI ${ }^{1) *}$ \\ ${ }^{1)}$ First Laboratory of Anatomy, Azabu University School of Veterinary Medicine, 1-17-71 Fuchinobe, Sagamihara, Kanagawa 252-5201, \\ Japan \\ 2) Zephyr Animal Hospital, 21-3 Namikicho, Hatiouji, Tokyo 193-0831, Japan \\ ${ }^{3}$ Seki Animal Hospital, 12 Minowacho, Seki, Gifu 501-3216, Japan \\ ${ }^{4)}$ Laboratory of Veterinary Radiology, Azabu University School of Veterinary Medicine, 1-17-71 Fuchinobe, Sagamihara, Kanagawa \\ 252-5201, Japan
}

(Received 5 May 2011/Accepted 23 August 2011/Published online in J-STAGE 6 September 2011)

\begin{abstract}
A simple method of lymphography of the thoracic duct was investigated. Using three female beagles, contrast media were administered rectally, vaginally and into the perianal tissue. The administration sites were gently massaged, and imaging was carried out at constant intervals using computed tomography and radiograph. Moreover, Indian ink was administered into the rectum mucous membrane in dogs for proof of this method of lymphography, and the lymph drainage routes were observed. The investigation showed that clear computed tomography and radiographic contrast images of the thoracic duct were obtained by subcutaneous and submucosa injection of angiography contrast medium and 3D processing of these images revealed the three-dimensional positions and course of the thoracic duct and cisterna chyli.
\end{abstract}

KEY WORDS: computed tomography, lymphography, radiograph, thoracic duct.

doi: 10.1292/jvms.11-0214; J. Vet. Med. Sci. 74(1): 135-140, 2012

Chylothorax is a condition in which chyle leaks into the thoracic cavity and accumulates. Symptoms include respiratory impairment, weight loss and exercise intolerance. In most cases, the etiology is unknown [4]. A number of medical and surgical treatments are currently suggested for the treatment of chylothorax. In many cases, because medical therapies are unable to resolve chylothorax completely, surgical treatment must ultimately be considered. To date, surgical treatment of chylothorax has included thoracic duct ligation [2], pleuroperitoneal shunting [11], thoracic omentalization [8], cisterna chyli ablation [6] and pericardiectomy [5], either alone or in combination with thoracic duct ligation. Thoracic duct ligation is recommended as the 1st choice among these methods $[3,5]$. One possible cause for the failure of thoracic duct ligation and subsequent recurrence is the occurrence mistakes in the surgery itself, such as failure to tie all of the complex tributaries into which the thoracic duct divides [1]. The thoracic duct in dogs is known to form either a single duct or multiple tributaries within the thoracic cavity after passing through the aortic hiatus, and their courses also display a complex morphology [7]. The position and course of the thoracic duct and cisterna chyli must be confirmed preoperatively when thoracic duct ligation or cisterna chyli ablation is performed, for which lymphography of the thoracic duct is of course

\footnotetext{
* Correspondence to: Asari, M., First Laboratory of Anatomy, Azabu University School of Veterinary Medicine, 1-17-71 Fuchinobe, Sagamihara, Kanagawa 252-5201, Japan. e-mail: asari@azabu-u.ac.jp
}

C2012 The Japanese Society of Veterinary Science required [1]. Current methods for performing lymphography of the thoracic duct involve the injection of contrast medium into the mesenteric lymph nodes during laparotomy under anesthesia or contrast imaging of the thoracic duct after the injection of contrast medium into the popliteal lymph node of a pelvic limb [2, 9, 10]. However, laparotomy is an invasive procedure, and reliable results cannot be obtained by percutaneous penetration of lymph nodes when the size and position of these lymph nodes are undetermined. In the present study, beagles were used to investigate new simple sites for administration of contrast media and for obtaining lymphographic images by computed tomography (CT) or radiography of the thoracic duct, which will have clinical applicability.

Three healthy female beagles (dogs A-C) weighing 8.4 $9.1 \mathrm{~kg}$ were used for CT imaging. Atropine $(0.025 \mathrm{mg} / \mathrm{kg}$ $\mathrm{sc})$ and butorphanol $(0.1 \mathrm{mg} / \mathrm{kg} \mathrm{sc})$ as anesthetic premedication and propofol (5.5-7.0 $\mathrm{mg} \mathrm{ml} / \mathrm{kg}$ iv) were administered to induce anesthesia. The trachea was intubated with isoflurane under $100 \%$ oxygen administration by inhalation to maintain anesthesia. Each anesthetized animal was placed in the supine position for CT imaging.

Using a 26-G 5/8-inch needle, warmed water-soluble contrast media (iopamidol, Iopamiron 370, Nihon Schering, Osaka, Japan) was injected into the ventral rectum mucous membrane of $\operatorname{dog} \mathrm{A}$ at a dose of $0.35 \mathrm{ml} / \mathrm{kg}$, into the ventral vagina mucous membrane of $\operatorname{dog} B$ at a dose of $0.35 \mathrm{ml} / \mathrm{kg}$ and into the subcutaneous tissue surrounding the anus of $\operatorname{dog} \mathrm{C}$ at a dose of $0.6 \mathrm{ml} / \mathrm{kg}$. Next, the administration sites were massaged gently for $5 \mathrm{~min}$, and imaging was carried 


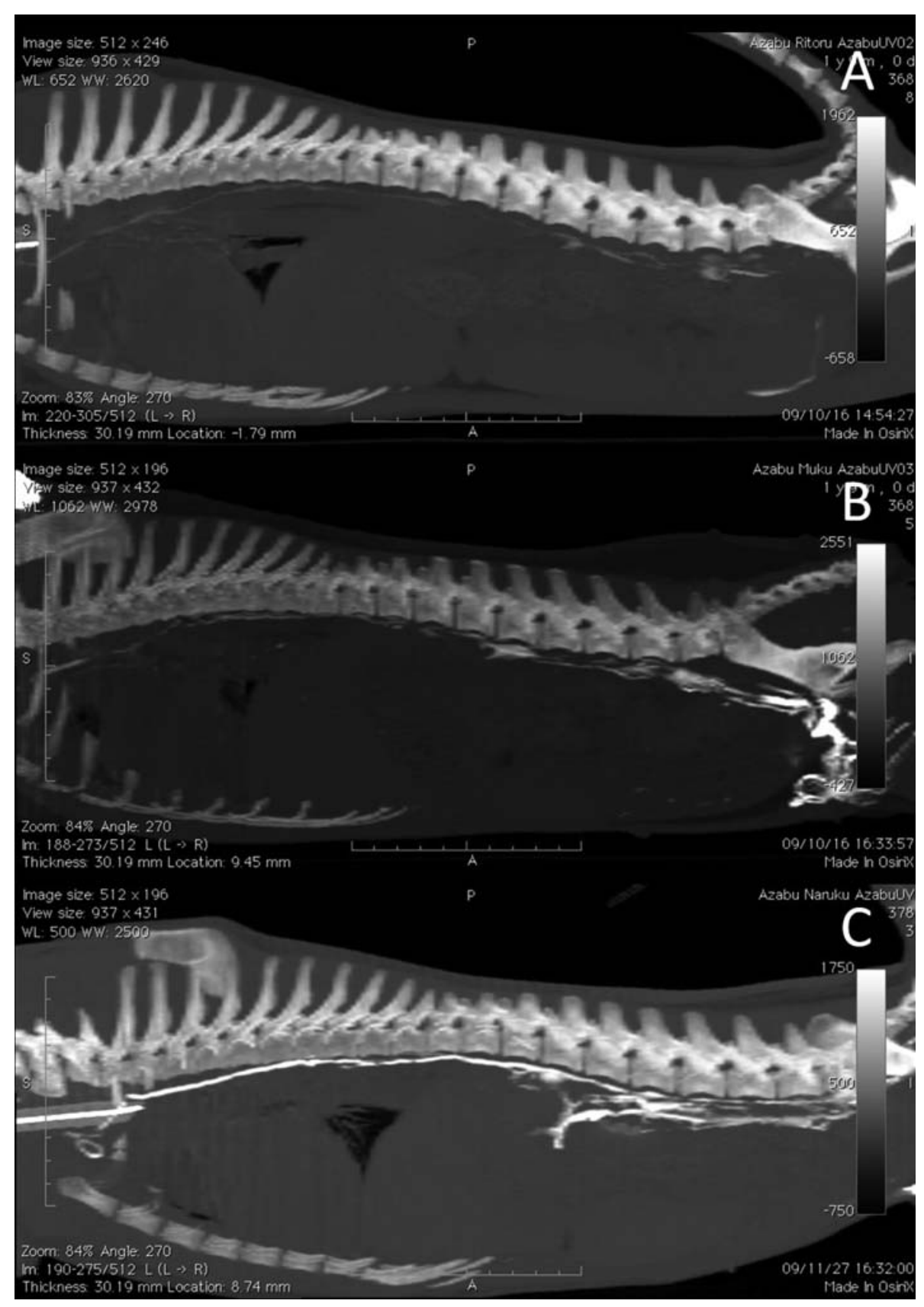

Fig. 1. Lateral CT images, thoracic duct lymphograms, after contrast media administration into the (A) rectum mucous membrane, (B) vagina mucous membrane (C) subcutaneous tissue surrounding the anus.

out $1,5,10,15$ and 30 min after massage. The image data acquired (DICOM data) were subjecter to $3 \mathrm{D}$ reconstruction using OsiriX version 2.7.5 (OsiriX Foundation, Geneva, Switzerland), to read the image in the DICOM files and export it to TIFF files. The CT scanner used in this experiment was a Toshiba Asteion TSX-021B single-slice device, and the imaging conditions were as follows: $120 \mathrm{kV} \mathrm{60-70}$ $\mathrm{mA}$ helical pitch, 1.5; scan speed, $0.75 \mathrm{sec}$; slice thickness,
$2 \mathrm{~mm}$; and reconstruction algorithm, FC80.

One healthy female beagle weighing $8.5 \mathrm{~kg}$ was used for radiographic imaging. After anesthetization, contrast media (iopamidol) was injected into subcutaneous tissue surrounding the anus. The dosage was $0.6 \mathrm{ml} / \mathrm{kg}$, and the area was massaged gently after administration for $5 \mathrm{~min}$; imaging was carried out 1,5 or $10 \mathrm{~min}$ after massage.

Moreover, to confirm the lymphatic vessels, anatomical 




Fig. 2. Lateral CT images, thoracic duct lymphograms, after contrast Series of transverse computed tomography (CT) images documenting the lymphatic drainage after subcutaneous administration of contrast medium (iopamidol) to the tissue surrounding the anus. (A) Transverse CT image at the level of the kidneys (LK, RK). The cysterna chyli is opacified (arrow). (B) Transverse CT image at the level of the 8th rib revealing 2 branches of the thoracic duct (arrows). (C) Transverse CT image at the level of the 5th rib. The thoracic ducts (arrow) change direction toward the left thoracic cavity, heading toward the vena cave.

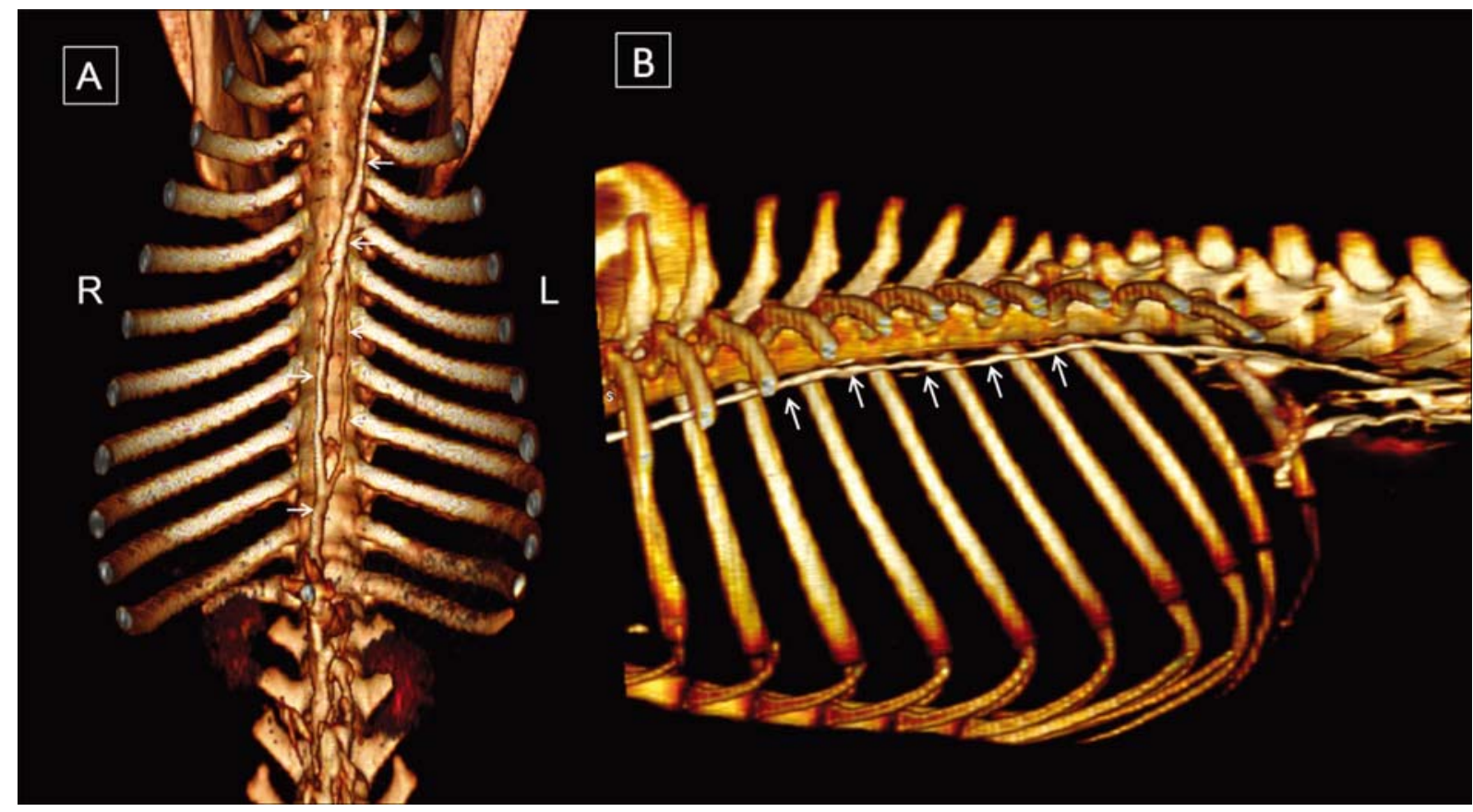

Fig. 3. Three-dimensional reconstruction from CT images of a thoracic duct lymphogram (A and B: lateral and ventrodorsal, respectively) 5 min after subcutaneous administration of contrast media to the tissue surrounding the anus (arrow: thoracic duct). Part of the rib was removed during processing.

observations were made using Indian ink. Two healthy beagles were used for this experiment. Warm Indian ink dissolved in physiological saline at a dose of $0.2 \mathrm{ml} / \mathrm{kg}$ body weight was injected into the ventral rectum mucous membrane of both dogs. Prior to the Indian ink injection, the dogs were given intramuscular injections of a mixture of 60 $\mu \mathrm{g} / \mathrm{kg}$ medetomidine hydrochloride (Domitor) and $2.5 \mathrm{mg} /$ $\mathrm{kg}$ ketamine hydrochloride to induce mild sedation. To accelerate arousal, the dogs were then given intramuscular injections of $160 \mu \mathrm{g} / \mathrm{kg}$ atipamezole hydrochloride to reverse the anesthesia, after which they were allowed to move freely in a paddock for $30 \mathrm{~min}$. They were then sacrificed by exsanguination under anesthesia with sodium pentobarbital and underwent whole-body retrograde fixation with ethylene glycol, liquefied phenol, formalin and methanol mixed in the ratio of $24: 8: 1: 16$ as a fixative solution. After complete fixation, the absorption fields and pathways of the drainage routes were observed macroscopically and under stereoscopic microscopy.

The animal facility at Azabu University has animal care 


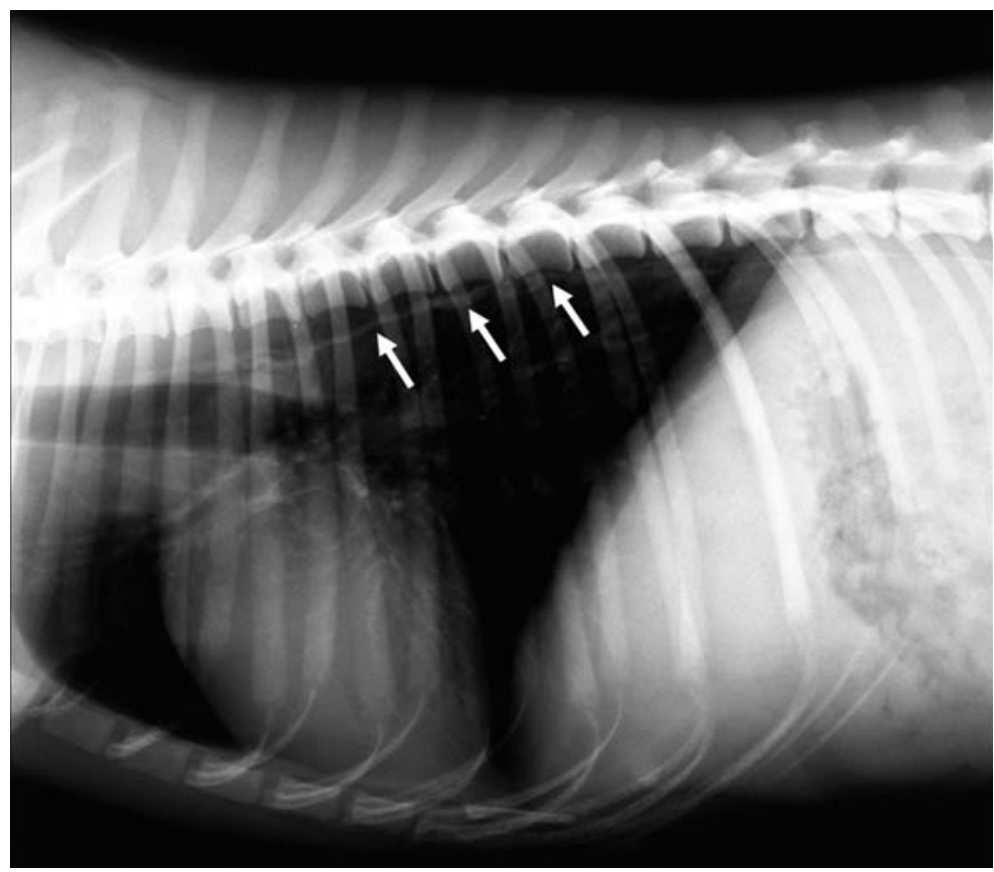

Fig. 4. The thoracic duct (arrows) is clearly visualized in a lateral thoracic radiogaraph 5 min after subcutaneous administration of contrast medium (iopamidol) to the tissue surrounding the anus.

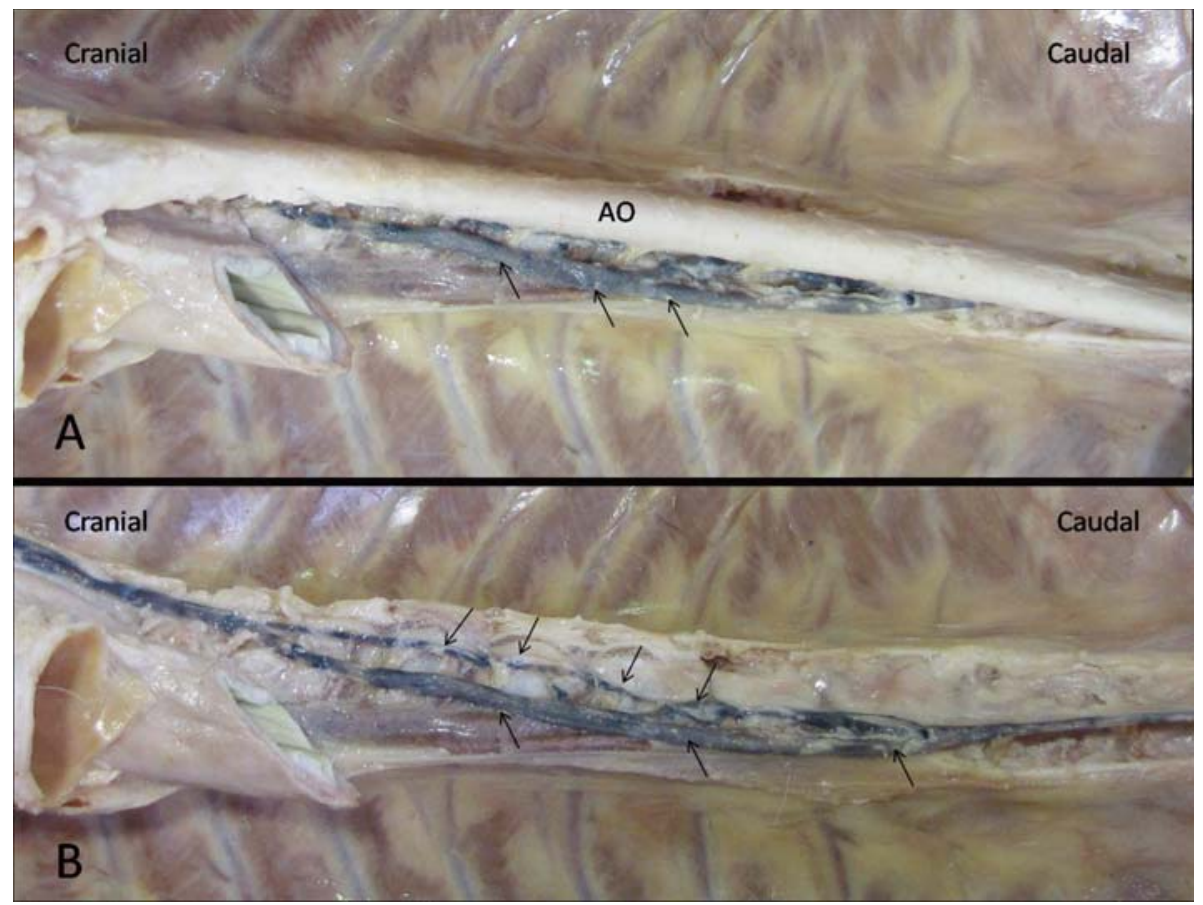

Fig. 5. Topographical photograph showing the thoracic cavity visualized with administration of indian ink beneath the rectal membranes. (A) The thoracic duct (arrows) is observed along the thoracic aorta (AO). (B) After the thoracic aorta is ablated, the fine tributaries of the thoracic duct (arrows) are observed. 
and use programs that are accredited by the Office of Laboratory Animal Welfare. All procedures in this study were in accordance with the guidelines approved by the Animal Research Committee of Azabu University.

The results for dogs A-C showed successful contrast observation of the contrast media passing through the lumbar lymphocenter and going through the diaphragm from the cisterna chyli to reach the thoracic duct (Fig. 1). After passing through the diaphragm, it was observed to run in a cranial direction slightly to the right of the midline of the thoracic cavity, but close to the vertebral body of the fifth thoracic vertebra, it was observed to change direction toward the left thoracic cavity, before which it branched into a number of fine tributaries. These tributaries were also depicted in detail by this method (Fig. 2). A comparison of results obtained $1,5,10,15$ and 60 min after the postadministration massage found that contrast results were best after 1 and $5 \mathrm{~min}$ for all administration sites, with thoracic duct images starting to disappear after $10 \mathrm{~min}$ had passed, at which point the contrast medium was being visibly excreted from the kidneys to the bladder.

CT imaging at $5 \mathrm{~min}$ after administration of contrast media to tissue surrounding the anus enabled the course of the thoracic duct to be depicted three-dimensionally (Fig. 3).

Similarly, the contrast images of the thoracic duct from radiographic imaging were best at 5 min after administration and massage of the tissue surrounding the anus (Fig. 4).

Anatomical observations after administration of Indian ink showed the development of a profuse lymphatic plexus under the rectum mucous membrane, with numerous fine lymph ducts leading from this lymphatic plexus to the lumbar lymphocenter, such as the medial iliac lymph nodes, and lymph ducts leading from the lumbar lymphocenter to the thoracic duct via the cisterna chyli (Fig. 5). These results revealed the fine tributaries of the thoracic duct in the same way as the results obtained from the CT images following interstitial injection of contrast medium.

In the present study, clear CT contrast images of the thoracic duct were obtained by the injection of water-soluble contrast media into the ventral rectum mucous membrane, the ventral vagina mucous membrane and subcutaneous tissue surrounding the anus, and $3 \mathrm{D}$ processing of these images revealed the three-dimensional positions and course of the thoracic duct and cisterna chyli. To achieve successful thoracic duct ligation, the 1st-choice treatment for chylothorax [3, 5], an accurate assessment of the thoracic duct is needed to determine the ligation site, and whether or not the thoracic duct can be clearly delineated is key to this process. Conventionally, the thoracic duct has been depicted by the direct administration of contrast medium into lymph nodes, but the comparatively well-known direct administration into the mesenteric root lymph nodes, for example, is highly invasive because it requires laparotomy [2]. Unlike conventional methods requiring procedural experience, in present method, it is possible to obtain a better understanding of individual differences in the thoracic duct more easily. Direct administration into the popliteal lymph node in a hind leg, which has recently been reported, is less invasive because it only involves injection into subcutaneous lymph nodes $[9,10]$, but these lymph nodes are small and mobile, meaning that a certain amount of experience is required for administration at this site. Unlike conventional methods requiring procedural experience, in the experiments in the present study, angiography contrast medium, used clinically on an everyday basis, was injected beneath the rectal membranes continuous with the anus and the vaginal membranes continuous with the vulva, as well as into perianal tissue, all sites that are easy to approach.

Of the rectum mucous membrane, the ventral vagina mucous membrane and the tissue surrounding the anus tested in this study, the area surrounding the anus was identified as the best site for administration (Fig. 1).

Choosing this site has several advantages: administration in the area surrounding the anus can be performed more easily than other sites, a higher dosage can be administered and massage can be performed easily because the administration site is on the surface. Massage was effective in promoting the lymph flow and allowed the thoracic duct to be described clearly.

In addition, 3D reconstruction of the $\mathrm{CT}$ images demonstrated both the three-dimensional morphology of the thoracic duct itself and its relationship to surrounding structures such as the thoracic vertebrae, ribs and thoracic aorta, while clearly depicting its course (Fig. 3). Additional experiments performed to verify these results by tracing the lymphatic ducts with Indian ink also confirmed the same flow. When the lymphatic ducts were traced with Indian ink, ink injected into the ventral rectum mucous membrane was clearly observed to reach the lymphatic plexus, while administration of contrast media to the ventral rectum mucous membrane resulted in it passing through the profuse lymphatic plexus and converging on the medial iliac lymph nodes, after which it passed through the thoracic duct via the cisterna chyli. The results indicate that when performing thoracic duct ligation and cisterna chyli ablation as surgical treatment for chylothorax, this method is more reliable and easier for depiction of the thoracic duct and cisterna chili. At three months after administration, no side effects were observed in the animals. However, the long term side effects and side effects of continuous use require further examination.

ACKNOWLEDGMENT. This work was supported by a Grant-in-Aid from the Japanese Society for Promotion of Science (No. 21580397).

\section{REFERENCES}

1. Billbery, S. A. and Birchard, S. J. 1994. Pulmonary lymphatics in dogs with experimentally induced chylothorax. J. Am. Anim. Hosp. Assoc. 30: 86-91.

2. Birchard, S. J., Cantwell, H. D. and Bright, R. M. 1982. Lymphangiography and ligation of the canine thoracic duct: a study in nomal dogs and three dogs with chylothorax. J. Am. Anim. Hosp. Assoc. 18: 769-777. 
3. Birchard, S. J. and McLoughlin, M. A. 1998. Treatment of idiopathic chylothorax in dogs and cats. J. Am. Anim. Hosp. Assoc. 212: 652-657.

4. Fossum, T. W. 1998. The characteristics and treatments of feline chylothorax. Comp. on. Cont. Ed. 914-928.

5. Fossum, T. W., Mertens, M. M., Miller, M. W., Peacock, J. T., Saunders, A., Gordon, S., Pahl, G., Makarski, L. A., Bahr, A. and Hobson, P. H. 2004. Thoracic duct ligation and pericardectomy for treatment of idiopathic chylothorax. J. Vet. Intern. Med. 18: 307-310.

6. Hayashi, K., Sicard, G., Gellasch, K., Frank, J. D., Hardie, R. J. and McAnulty, JF. 2005. Cisterna chyli ablation with thoracic duct ligation for chylothorax:result in eight dogs. Vet. Surg. 34: 519-523.

7. Kagan, K. G. and Breznock, E. M. 1979. Variation in the canine thoracic duct system and the effects of surgical occlusion demonstrated by rapid aqueous lymphography, using an intestinal lymphatic trunk. Am. J. Vet. Res. 40: 948-958.
8. LaFond, E., Weirich, W. E. and Salisbury, S. K. 2002. Omentalization of the thorax for treatment of idiopathic chylothorax with constrictive pleuritis in a cat. J. Am. Anim. Hosp. Assoc. 38: 74-78.

9. Millward, I.R., Kirberger, R.M. and Thompson, P.N. 2011 Comparative popliteal and mesenteric computed tomography lymphangiography of the canine thoracic duct. Vet. Radiol. Ultrasound. 52: 295-301.

10. Naganobu, K., Ohigashi, Y., Akiyoshi, T., Hagio, M., Miyamoto, T. and Yamaguchi, R. 2006. Lymphography of the thoracic duct by percutaneous injection of iohexol into the popliteal lymph node of dogs:experimental study and clinical application. Vet. Surg. 35: 377-381.

11. Smeak, D. D., Stephenj., Birchard., McLoughlin, M. A., Lindsey, M. M., Holt, D. E., Caywood, D. D. and Downs, M. O. 2001. Treatment of chronic pleural effusion with pleuroperitoneal shunts in dogs 14 cases (1985-1999). J. Am. Vet. Med. Assoc. 219: 1590-1597. 